PALABRAS CLAVE | OCÉANO | PLÁSTICO | CONTAMINACIÓN | DESCONTAMINACIÓN | DIALIZACIÓN

KEYWORDS I OCEAN I PLASTIC | POLLUTION

DECONTAMINATIONIDIALIZATION

\section{Oceanic Plastic Filter Platform}

\section{| RESUMEN}

La propuesta plantea una solución arquitectónica para enfrentar los daños causados por la basura de plástico que flota en el océano como resultado de muchos años de cultura desechable. En este contexto, el proyecto fue creado como un prototipo de una plataforma flotante que filtran el océano, capturando y absorbiendo el plástico flotante sin afectar a la biodiversidad. El proyecto, que se localiza a 4 kilómetros de la costa de la Isla de Pascua, cerca del centro del megavórtice del Océano Pacífico Sur, un punto estratégico para comenzar una limpieza mundial y convertirse en un estándar ecológico.

\section{| ABSTRACT |}

The project offers an architectonical solution to the damage caused to the environment by plastic garbage floating in the ocean as a result of many years of throwaway culture. In this context, the project was developed as a prototype of a Floating Platform that filters the ocean, capturing and absorbing the floating plastic without affecting the biodiversity. The project is located $4 \mathrm{~km}$ off the coast of Easter Island, near the center of the mega-vortex of the South Pacific Ocean, home to millions of tons of floating plastic; thus a strategic point to start a global clean up and turn into a worldwide ecological standard.

\title{
Plataforma de dialización oceánica Proyecto para la descontaminación de plástico en los océanos
}

U de los rasgos esenciales de la cultura contemporánea es su tendencia hacia lo desechable, cuestión que ha traído como consecuencia un fuerte impacto sobre el ecosistema y sus recursos naturales. En este contexto, la propuesta arquitectónica aquí presentada aborda la contaminación generada por residuos plásticos en los océanos.

A partir de la década de los 60 del siglo pasado, el plástico pasó a ser parte de nuestra vida cotidiana, permitiéndonos, entre otras cosas, alargar nuestra esperanza de vida. Sin embargo, más allá de los beneficios de un material extremadamente durable, se trata de un producto biodegradable en 500 años, diseñado para un uso de 5 minutos promedio y que pocos saben dónde va a parar esta gran masa de basura: a los océanos, matando y destruyendo de manera silenciosa, incluso a nosotros.

Hoy en día existen seis megavórtices de plástico flotantes, cinco entre continentes y un sexto cerca del Ártico, siendo el más grande el del Pacífico Norte, con un tamaño similar al de Brasil $(8,5$ millones de $\mathrm{km}^{2}$ ), con un espesor de 10 metros, hogar perfecto para el Halobates (insecto que ha tenido crecimiento exponencial descontrolado y se alimenta de zooplancton, eliminando la base de la cadena alimenticia).

En este contexto, la propuesta constituye un prototipo de plataforma flotante que filtra el océano, capturando y absorbiendo el plástico situado a 4 km, frente a las costas de Rapa Nui. El lugar de intervención constituye un espacio estratégico para comenzar la limpieza global de océanos, debido a que se localiza en el centro del megavórtice del Pacífico Sur donde toneladas de microbasura plástica llegan en cada ola. Así, una propuesta adecuada podrá convertirse en un referente mundial de la nueva ecología.

En términos formales, el proyecto fue concebido horizontalmente, lo que se traduce en un volumen no superior a un barco carguero, quedando así la zona de programa habitable en la superficie y la zona de reciclaje hundida, respetando el paisaje natural visto desde Rapa Nui. Por otro lado, el hundimiento parcial de la plataforma genera una verticalidad natural, por lo cual el programa se distribuye en torno a un gran espacio articulador (plaza vertical) que permite una vinculación directa entre el cielo y el agua y viceversa. 
1. Vista aérea.

2. Plataforma.

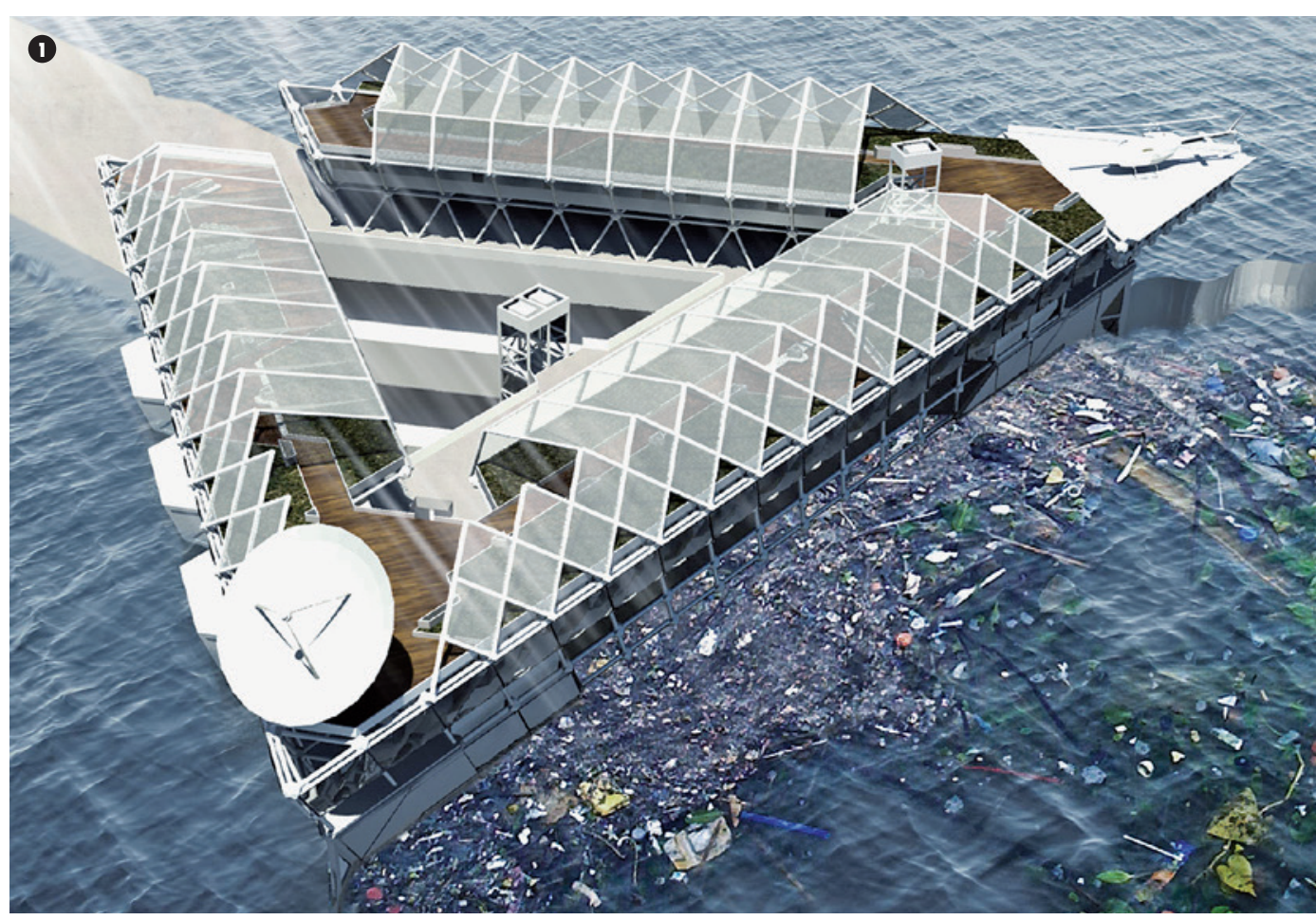

2

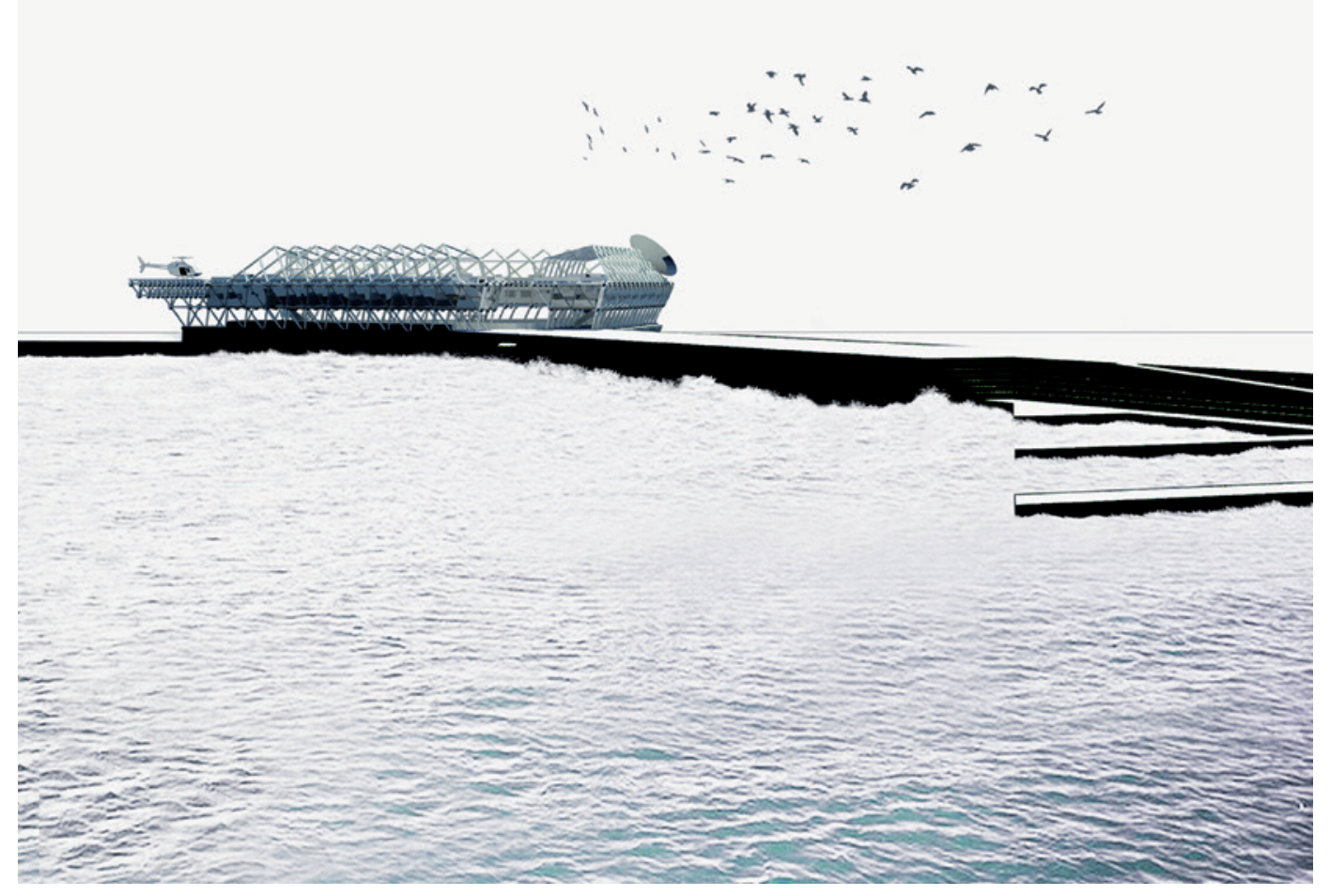

Otra característica importante es que la plataforma es capaz de generar su propia energía, a través de los brazos de captación y comida en sus huertos. Así, el objeto arquitectónico aprovecha su hundimiento para precipitar el océano en su interior, directo a la zona de reciclaje, utilizando filtros de gravedad que permiten separar el agua del plástico, para luego ser reciclado, obteniendo un ladrillo plástico que ha de ser utilizado para construcciones sociales. Sobre la zona habitable se encuentran los ya mencionados huertos para producir comida y no desabastecer la isla (considerando que trabajan 65 personas). Sobre esta hay un techo formado de celdas fotovoltaicas.

En cuanto al diseño estructural, este está basado en el fractal de Sierpinski, pero pensado en tres dimensiones, con el fin de lograr la coordinación total de la estructura con elementos pequeños y prefabricables, logrando un tetraedro simple, limpio, involcable, indeformable y estático. Para capturar el plástico y atraerlo a la plataforma se diseñó un sistema modular de barreras ondulantes que utilizan el oleaje para separar lo vivo de lo inerte al mismo tiempo que producen energía, estas pueden replicarse por la longitud que se necesite, ya que deja el libre paso de peces, embarcaciones, etc., y así necesitar solo una plataforma cada varios kilómetros lineales, esto principalmente debido a los colosales tamaños de las corrientes oceánicas. El océano es dinámico, se mueve, por lo que no es necesario que la plataforma lo haga, esto significa que eventualmente toda el agua va a pasar por la misma zona, por lo que la disposición de una serie de plataformas funcionando sistemáticamente y abarcando cientos de kilómetros lineales, sería una buena manera de comenzar a arreglar el desastre que todos hemos hecho. 


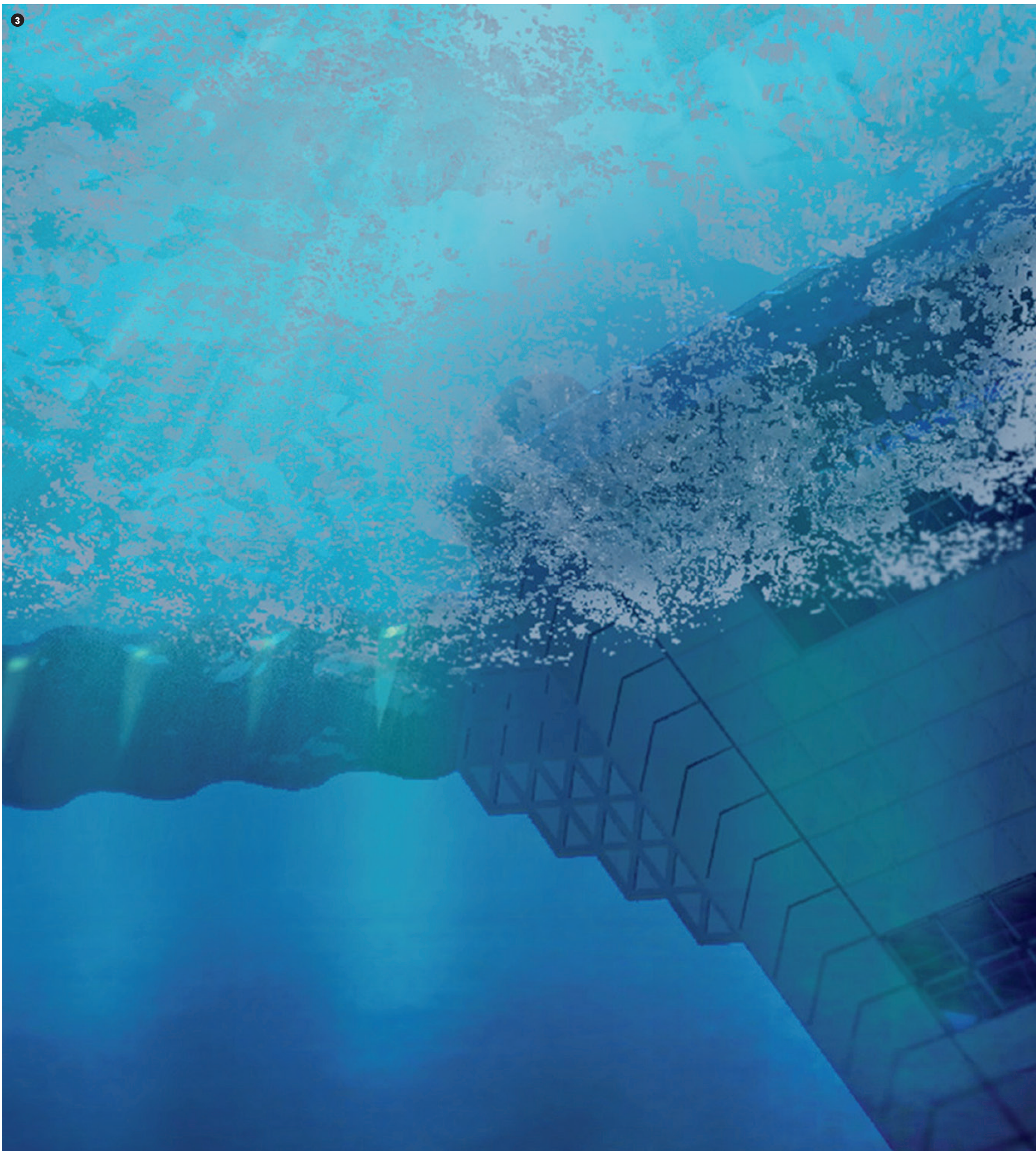


4. Despiece plataforma dialización oceánica.

5. Sistema estructural.

(4)
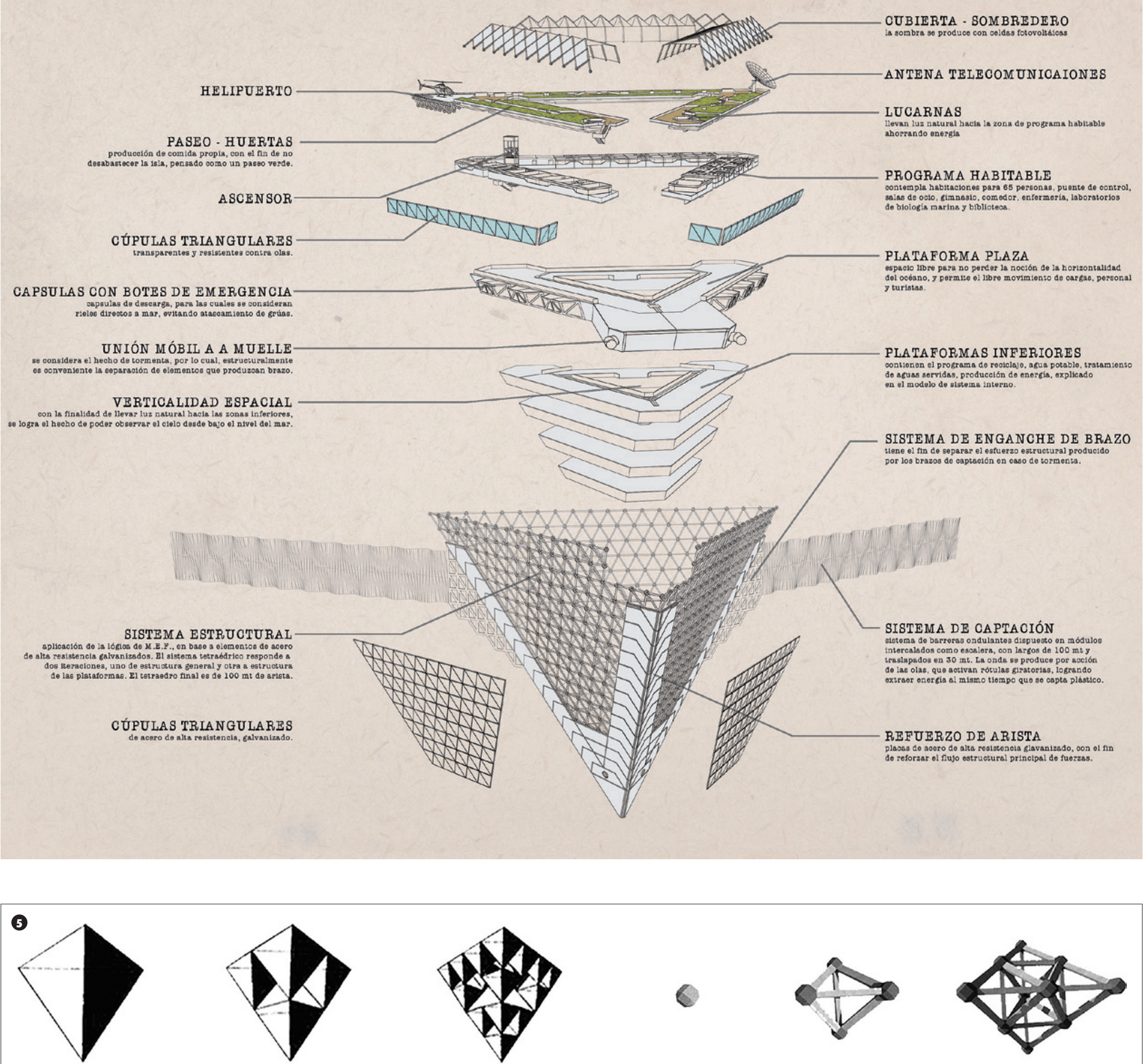
6. Planta.

7. Corte AA.

8. Despiece plataforma dialización oceánica.

○

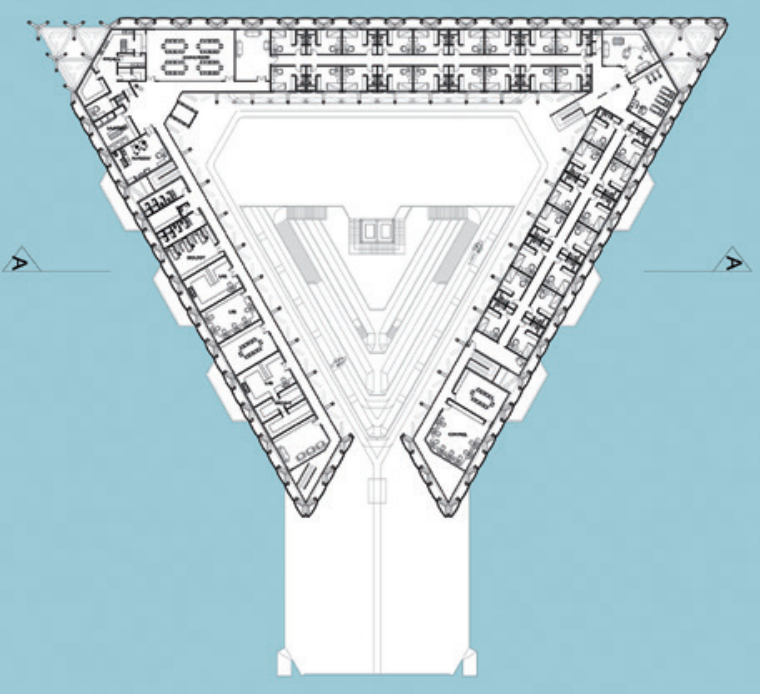

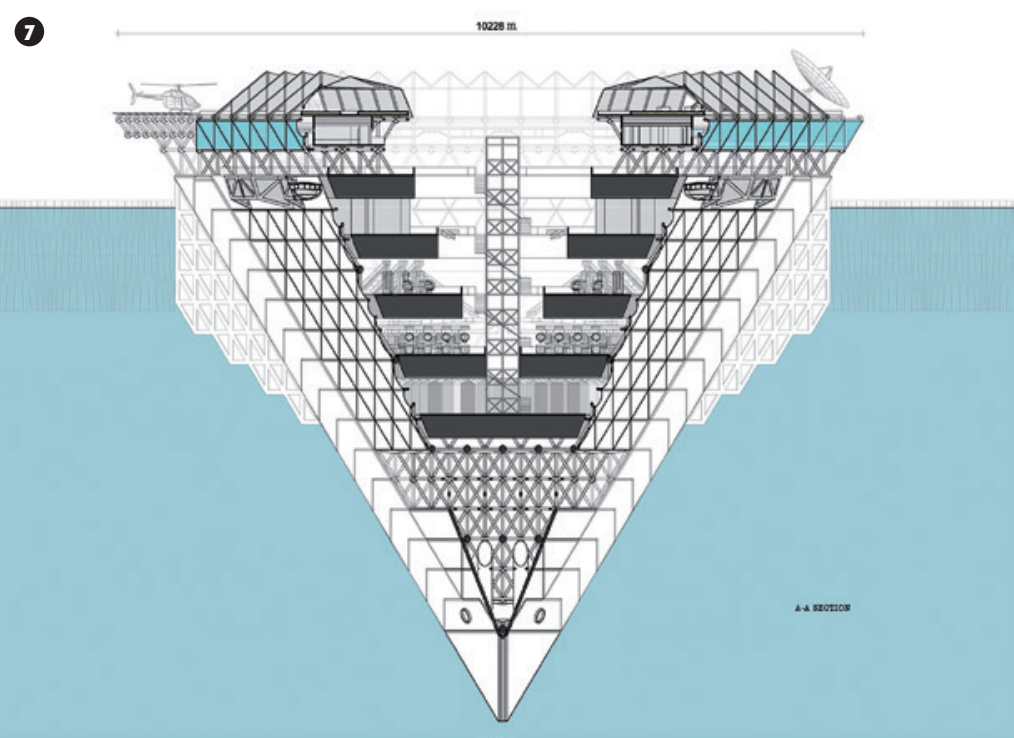

8

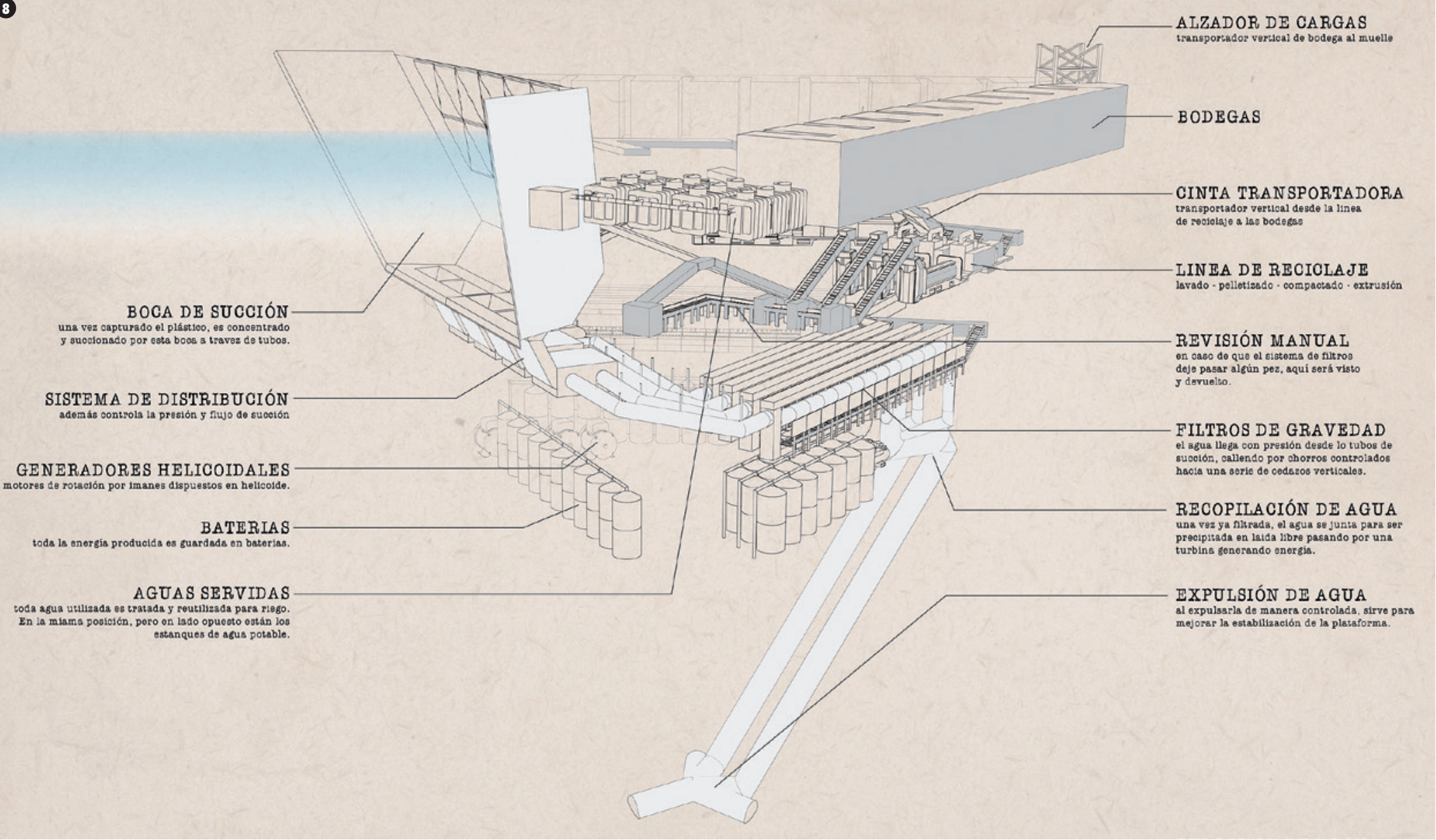




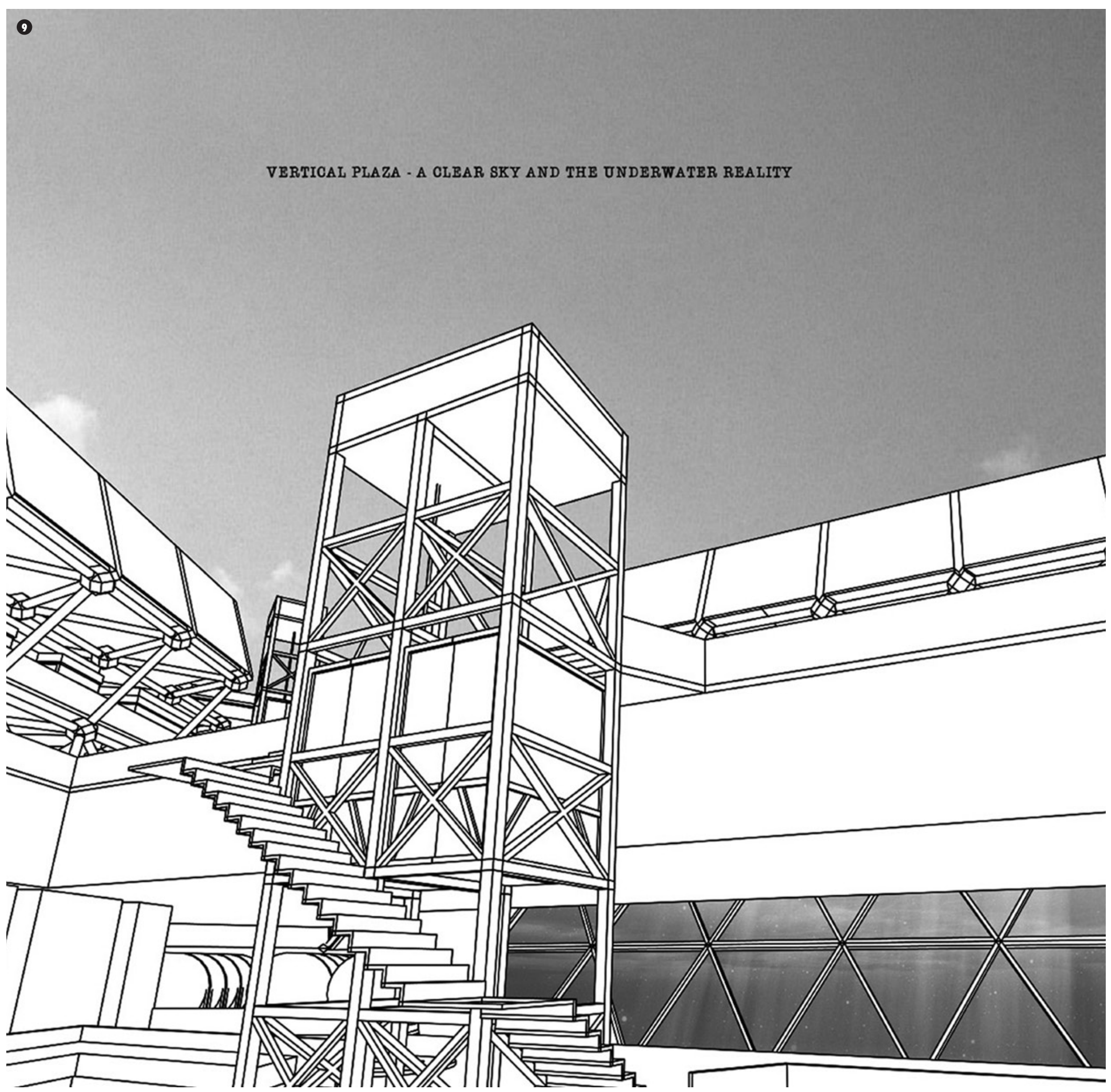

